\section{Deep sclerectomy and trabeculectomy in uveitic glaucoma}

\begin{abstract}
Purpose To compare the results of deep sclerectomy (DS) and trabeculectomy (TRAB) in the management of uveitic glaucoma. Methods A retrospective chart review was carried out of 41 eyes (38 patients) operated on for uncontrolled uveitic glaucoma, 20 by DS with an implant and 21 by TRAB, with antiproliferative agents in both cases. Main outcome criteria were intraocular pressure (IOP), flare values, complications, and need for postoperative adjustments or re-operation. Results Mean follow-up lasted 21.1 \pm 11.3 months in the TRAB group and 18.9 \pm 10.7 months in the DS group $(P=0.184)$. Mean IOP did not differ significantly in the two groups on the day before surgery, at postoperative days 1 and 7 , or at months 1, 6, and 12. At postoperative month 12 , IOP values in the TRAB and DS groups were $12.4 \pm 4.3$ and $11.7 \pm 3.3 \mathrm{~mm} \mathrm{Hg}(P=0.688)$, respectively. The cumulative probability of success at $\mathbf{1 2}$ months was $89 \%$ for TRAB and $88 \%$ for DS $(P=0.306)$. After DS, more postoperative adjustments were necessary to lower IOP than after TRAB (85 vs $9.5 \%, P<0.001$ ), but 7 days after surgery, intraocular inflammation was higher after TRAB (245.8 vs $38.5 \mathrm{ph} / \mathrm{ms}, P<0.001$ ). Conclusions DS and TRAB afforded equivalent IOP control in uveitic glaucoma. TRAB involved deeper invasion of the eye and resulted in more inflammation during the week after surgery, but successful DS required closer postoperative monitoring and more postoperative adjustments. Eye (2010) 24, 310-314; doi:10.1038/eye.2009.82; published online 17 April 2009
\end{abstract}

Keywords: uveitis; glaucoma; trabeculectomy; deep sclerectomy

\section{Introduction}

The surgical management of uveitic glaucoma refractory to medical therapy is complex and
B Dupas, C Fardeau, N Cassoux, B Bodaghi and $\mathrm{P}$ LeHoang

challenging. The results of filtering surgery, even with a draining device and antiproliferative agents, vary greatly, and the failure rate is higher for uveitic than non-uveitic glaucoma. ${ }^{1-3}$ Ten years ago, deep sclerectomy (DS), a non-penetrating surgical operation, was designed to lower the incidence of complications after trabeculectomy (TRAB). ${ }^{4}$ DS consists of the physical removal of the resisting element without endangering the eye's structural integrity. The main resistance to aqueous egress lies in the juxtacanalicular trabeculum and inner wall of Schlemm's canal. The DS procedure induces selective filtration through the trabeculodescemetic membrane and evacuation of the aqueous from the intrascleral space. In primary open-angle glaucoma, both DS with a collagen implant and TRAB result in equal control of intraocular pressure (IOP), and similar cumulative success rates have indeed been reported for the two techniques. ${ }^{5,6}$ In addition, DS seems to require fewer postoperative complications than TRAB, although results for the longevity of IOP stabilisation remain uncertain. ${ }^{7}$ Few complications and low levels of postoperative intraocular inflammation make DS a surgical procedure of interest in the management of uveitic glaucoma. However, because DS surgery is non-penetrating, it may fail to control long-term IOP in eyes exhibiting inflammatory changes in the trabecular meshwork. Both these types of filtering surgeries (DS and TRAB) have been evaluated in uveitic glaucoma, but separately. ${ }^{1,2,8-11}$ In this retrospective study, we report the mid-term results of both TRAB and DS with antiproliferative agents, in eyes with uveitic glaucoma.

\section{Patients and methods}

We retrospectively reviewed the medical files of 38 consecutive patients who underwent filtering surgery for medically uncontrolled uveitic glaucoma at the Pitié Salpétrière Hospital in 
Paris, France (a tertiary uveitis center), between November 2003 and February 2005. As it was a retrospective chart review, no IRB (CPP) approval was required to approve the review of the medical records. The study and data accumulation were carried out in conformity with French and European laws, and in adherence to the tenets of the Declaration of Helsinski. Oral informed consent was obtained from the patients for the review of their data. Exclusion criteria were inflammation unrelated to uveitis, eye surgery during the 6 months before filtering surgery, and incomplete medical records. The patients operated on had medically uncontrolled uveitic glaucoma, defined as an IOP higher than $21 \mathrm{~mm} \mathrm{Hg}$ measured under maximal medical therapy, with no peripheral anterior synechiae after gonioscopic examination, and inflammation controlled medically with local and/or systemic agents (steroids, or immunomodulatory or anti-infectious therapy) at the time of surgery. Individuals had not been assigned to any particular surgical technique, as patients had been recruited serially by an expert in each technique. Consequently, only one surgeon carried out each type of surgery (PLH for DS and CF for TRAB). The DS was carried out with intraoperative mitomycin $C$ in a concentration of $0.4 \mathrm{mg} / \mathrm{ml}$ for an average time of 3 min under the Tenon capsule, and a highly hydrophilic acrylic implant (T-flux, Ioltech, La Rochelle, France) was placed radially and secured with a single 10-0 nylon suture. TRAB was carried out with intraoperative mitomycin $\mathrm{C}$ at the dose and application time described above for DS. Pre- and postoperative data were collected for the following: sex, age at initial surgery, duration of glaucoma before surgery, type of uveitis, lens status, earlier filtering surgery, best corrected visual acuity measured on the decimal scale and converted on a Snellen chart, IOP (measured using a Goldmann applanation tonometer, Haag-Streit, Bern, Switzerland), and flare values, measured with a laser flare-cell photometer (Kowa, Tokyo, Japan), and expressed in photons per millisecond ( $\mathrm{ph} / \mathrm{ms}$ ). All these data were derived from the results of examinations conducted on the first and seventh days after surgery, and 1, 6 and 12 months thereafter. For DS and TRAB, success was defined as a postoperative IOP of $21 \mathrm{~mm} \mathrm{Hg}$ or lower, with or without the use of IOPlowering agents, and failure, as an IOP greater than $21 \mathrm{~mm} \mathrm{Hg}$ with the use of medication, loss of light perception, or the need for additional surgery. If a goniopuncture, needling with antimitotic injection, or flap suture modification was necessary to control IOP; the case was considered a success if one of these procedures lowered IOP to $<21 \mathrm{~mm} \mathrm{Hg}$. The criteria for undertaking such postoperative adjustments were used, depending on each surgeon's clinical judgement, as follows. For TRAB, IOP values $\geqslant 21$ were managed by IOP-lowering medications or by flap suture modification, if needed, and an IOP $\geqslant 28 \mathrm{~mm} \mathrm{Hg}$ was considered an indication for re-operation. For DS and IOP values $\geqslant 21 \mathrm{~mm} \mathrm{Hg}$, needling was carried out in case of encapsulated bleb; otherwise, goniopuncture was carried out. IOP-lowering medications were added only after postoperative adjustments, if needed. An IOP $\geqslant 28 \mathrm{~mm} \mathrm{Hg}$ was considered an indication for re-operation.

\section{Statistical analysis}

The qualitative and quantitative variables tested included patients' baseline characteristics and the results of the ophthalmic examinations listed above for the two groups of patients who underwent DS and TRAB, respectively. Depending on whether or not the variables conformed to a normal law, we applied parametric or non-parametric tests. Qualitative variables were tested using the $\chi^{2}$-test or Fisher's exact test, and quantitative variables, using $t$-test or the Mann-Whitney $U$ test. $P$-values $<0.05$ were considered significant. The cumulative probability of success was analysed using Kaplan-Meier survival curves, and survival curves were compared between groups with the log-rank test.

\section{Results}

Of the 41 eyes treated, 21 underwent TRAB, and 20 underwent DS. Patients' demographic and historical data are shown in Table 1. The mean follow-up period was $21 \pm 11.31$ months in the TRAB group and $18.9 \pm 10.7$ months in the DS group $(P=0.184)$. Diagnoses of uveitis among individuals did not differ in the two groups and the leading causes were idiopathic inflammation and Fuchs heterochromic iridocyclitis. A total of 16 of the 21 eyes in the TRAB group and 14 of the 20 in the DS group completed 12 months of follow-up. The cumulative probability of success at 12 months was $89 \%$ for TRAB and $88 \%$ for DS ( $P=0.306$, log-rank test, Figure 1$)$. Figure 2 shows the evolution of IOP over time in both groups, and Table 2 gives detailed IOP values. Mean preoperative IOP \pm SD was $30.2 \pm 6.7 \mathrm{~mm} \mathrm{Hg}$ for the TRAB group, and $29.2 \pm 7 \mathrm{~mm} \mathrm{Hg}$ for the DS group $(P=0.832)$. There were no significant intergroup differences between mean postoperative IOP at day 7 or at months 1, 6 and 12. Mean IOP at 12 months was $12.4 \pm 4.3 \mathrm{~mm} \mathrm{Hg}$ for the TRAB group and $11.7 \pm 3.3 \mathrm{~mm} \mathrm{Hg}$ for the DS group $(P=0.688)$. Mean preoperative intraocular flare values were also similar in both groups (Table 2). On the other hand, a significantly greater increase in intraocular inflammation was 
Table 1 Summary of patients' demographics and history

\begin{tabular}{lccr}
\hline & Trabeculectomy & Deep sclerectomy & P \\
\hline Eyes, $n$ & 21 & 20 & - \\
Age at surgery (years), mean \pm SD (range) & $44.9 \pm 23.1(7.6-78.4)$ & $40.9 \pm 12(16.9-62.1)$ & 0.201 \\
Gender, M/F & $8 / 13$ & 4.5 & 0.762 \\
Duration of glaucoma, years & 4.6 & 7 & 0.349 \\
Earlier cataract surgery, $n$ & 10 & $18.9 \pm 10.7$ & 0.412 \\
Postoperative follow-up (months), mean \pm SD & $21.1 \pm 11.3$ & 1 & 0.184 \\
Total no. of earlier filtering surgeries, $n$ & 5 & 8 & 0.088 \\
& & 4 & 0.141 \\
Diagnosis of uveitis, $n$ & & 1 & 0.335 \\
Fuchs heterochromic iridocyclitis & 4 & 2 & 0.089 \\
Idiopathic inflammation & 7 & 0 & 0.520 \\
Juvenile idiopathic arthritis (JIA) & 5 & 2 & 0.079 \\
Viral (CMV, HSV) & 1 & 3 & 0.137 \\
Sarcoïdosis & 3 & 0.269 \\
Tuberculosis & 0 & 1 & \\
Others & & & \\
\hline
\end{tabular}

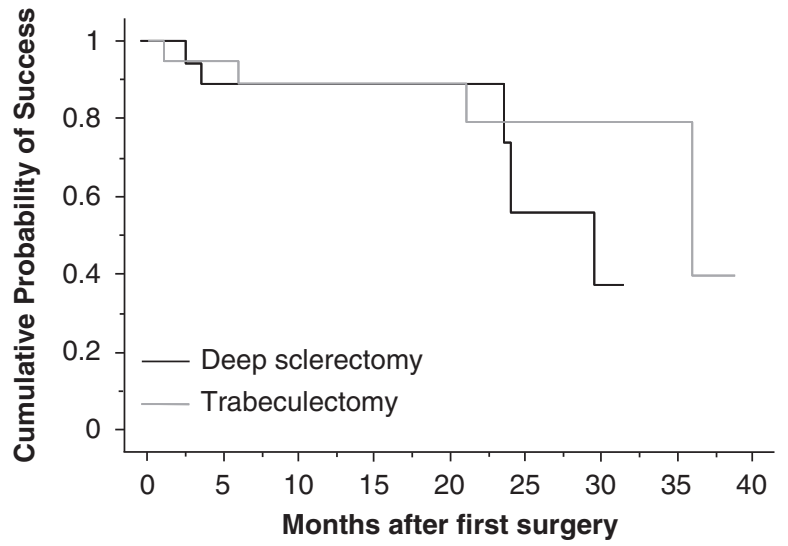

Figure 1 Cumulative probability of success over time after initial surgery (deep sclerectomy or trabeculectomy).

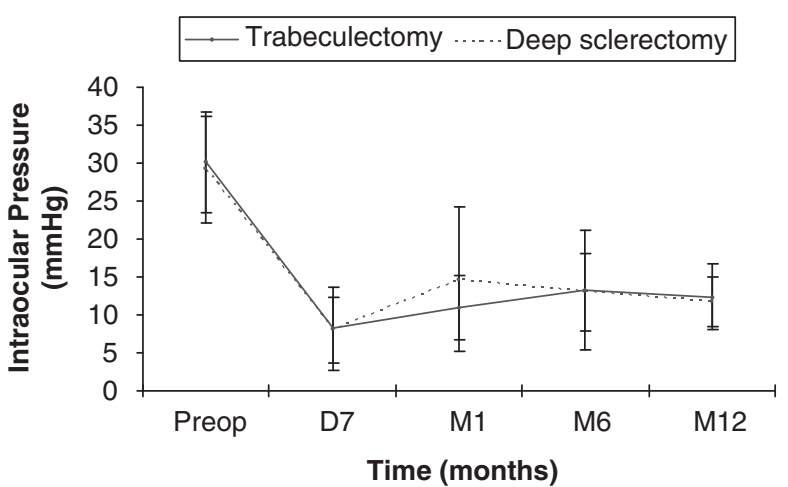

Figure 2 Intraocular pressure over time, before and after deep sclerectomy or trabeculectomy.

observed at postoperative day 7 in the TRAB than in the DS group (245.8 ph/ms (16.9-1200) vs $38.5 \mathrm{ph} / \mathrm{ms}$ (4.8-78), $P<0.001)$.
Table 2 Preoperative and postoperative eyes status after trabeculectomy and deep sclerectomy

\begin{tabular}{|c|c|c|c|}
\hline & Trabeculectomy & $\begin{array}{c}\text { Deep } \\
\text { sclerectomy }\end{array}$ & $\mathrm{P}$ \\
\hline $\begin{array}{l}\text { Mean preoperative } \\
\text { IOP } \pm \text { SD }\end{array}$ & $30.2 \pm 6.7(21)$ & $29.2 \pm 7(20)$ & 0.832 \\
\hline \multicolumn{4}{|c|}{ Mean postoperative IOP $\pm S D$} \\
\hline At day 7 & $8.3 \pm 5.5(18)$ & $8 \pm 4.3(20)$ & 0.863 \\
\hline At month 1 & $11 \pm 4.2(20)$ & $14.7 \pm 9.6(18)$ & 0.890 \\
\hline At month 6 & $13.3 \pm 7.9(19)$ & $13 \pm 5.1$ & 0.713 \\
\hline At month 12 & $12.4 \pm 4.3(16)$ & $11.7 \pm 3.3(14)$ & 0.688 \\
\hline \multicolumn{4}{|c|}{ Preoperative visual acuity, $\mathrm{n}$} \\
\hline$<20 / 40$ & 9 & 8 & 0.853 \\
\hline$\geqslant 20 / 40$ & 11 & 12 & 0.623 \\
\hline \multicolumn{4}{|c|}{ Postoperative visual acuity, $\mathrm{n}$} \\
\hline$<20 / 40$ & 9 & 6 & 0.393 \\
\hline$\geqslant 20 / 40$ & 11 & 13 & 0.412 \\
\hline \multicolumn{4}{|c|}{ Mean flare measurements (photons/ms) } \\
\hline Preoperative & $52.8(15)$ & $32(14)$ & 0.057 \\
\hline Postoperative day 7 & $245.8(6)$ & $38.5(13)$ & $<0.001$ \\
\hline Month 6 & $30.3(12)$ & $26.2(7)$ & 0.431 \\
\hline
\end{tabular}

Numbers in parenthesis indicate number of eyes.

Table 3 shows the complications that occurred during follow-up, and the postoperative adjustments and re-operations carried out during that period. A total of 5 of the 21 eyes in the TRAB group underwent surgery for uncontrolled IOP, three underwent repeat TRAB (in all three, IOP dropped to below $21 \mathrm{~mm} \mathrm{Hg}$ ), and two eyes underwent laser diode ciliary photocoagulation for high uncontrolled IOP. Postoperative adjustments were only carried out in 2 of the 21 eyes (9.5\%): one had laser scleral flap suture lysis, and the other, scleral flap suture. 
Table 3 Complications, postoperative adjustments and re-operations

\begin{tabular}{lccc}
\hline & $\begin{array}{c}\text { Trabeculectomy } \\
(\mathrm{n}=21)\end{array}$ & $\begin{array}{c}\text { Deep } \\
\text { sclerectomy } \\
(\mathrm{n}=20)\end{array}$ & $\mathrm{P}$ \\
\hline Complications & $n=2$ & $n=1$ & 0.519 \\
$\quad \begin{array}{l}\text { Hypotony } \\
\text { Hyphema }\end{array}$ & 2 & 0 & \\
& 0 & 1 & \\
Postoperative adjustments & $n=2$ & $n=17$ & $<0.001$ \\
Needling & 0 & 3 & \\
Goniopuncture & 0 & 9 & \\
Scleral flap or conjunctival & 2 & 5 & \\
flap suture lysis or closure & & & \\
& $n=5$ & $n=4$ & 0.645 \\
Re-operation & 3 & 4 & \\
$\quad$ Trabeculectomy & 2 & 0 & \\
Ciliary photocoagulation & & & \\
\hline
\end{tabular}

In the DS group, the number of postoperative adjustments was much larger (17/20 eyes, $85 \%$, $P<0.001)$. In this group, 9 out of 20 eyes (45\%) underwent a goniopuncture that lowered IOP to below $21 \mathrm{~mm} \mathrm{Hg}$ in all cases. In addition, three eyes (15\%) underwent needling, three (15\%) conjunctival flap suture for bleb leakage, and two (10\%) laser scleral flap suture lysis. In all the cases, these postoperative adjustments lowered IOP to below $21 \mathrm{~mm} \mathrm{Hg}$. As regards re-operation, four eyes in the DS group required TRAB, after which IOP dropped to $<21 \mathrm{~mm} \mathrm{Hg}$ in three cases.

Postoperative complication rates were not different in the two groups $(P=0.519)$. During postoperative follow-up, 4 of the 11 eyes in the TRAB group (36.3\%) underwent phacoemulsification with posterior chamber intraocular lens implantation, vs 6 out of $13(46 \%)$ in the DS group, with good control of IOP and uveitis $(P=0.350)$. Lastly, mean visual acuity, preoperatively and 6 months postoperatively, was not different in the two groups (Table 2).

\section{Discussion}

Our study of the surgical management of uveitic glaucoma showed that similar mid-term control of IOP was obtained by either TRAB or DS, with similar success rates at 12 months. No significant difference between the results of these procedures was found for postoperative complications or the need for re-operation. However, to lower IOP to below $21 \mathrm{~mm} \mathrm{Hg}$, DS required many more postoperative adjustments than TRAB, but TRAB induced marked, although transient, worsening of intraocular inflammation. Visual acuity scores and postoperative cataract progression requiring phacoemulsification were similar in both the groups.
In other studies of the management of uveitic glaucoma, previous authors compared the results of different surgical procedures, including the use of the Ahmed valve and Molteno implant, goniosurgery, or viscocanalostomy. ${ }^{1,3,12-14}$ In many studies, the results of DS and TRAB were also compared in patients with primary glaucoma. ${ }^{5-7,15,16}$ However, as regards the management of uveitic glaucoma, only one of these two procedures was usually analysed., ${ }^{2,9-11}$ For TRAB, our surgical success rate of $89 \%$ at 12 months is comparable with the rates reported by Ceballos ${ }^{1}$ or Towler ${ }^{2}$ for uveitic glaucomas. This rate is slightly lower than the $90 \%$ obtained at 2 years by Ambresin ${ }^{15}$ after TRAB in non-uveitic glaucoma, but our $88 \%$ success rate for DS is not different from the $88 \%$ reported at 12 months by Souissi, ${ }^{17}$ and is only slightly lower than that reported by Auer $^{9}$ (90\% at 1 year, in a cohort of 14 eyes). As expected, this success rate for DS is also lower than the one obtained for non-uveitic glaucomas by Shaarawy ${ }^{18}$ ( $100 \%$ at 48 months). Here, in DS-treated eyes, the success rate of $88 \%$ was obtained thanks to numerous postoperative interventions designed to lower IOP, including goniopuncture in $45 \%$ of the cases and needling in $15 \%$, whereas in eyes treated by TRAB, postoperative adjustments were only carried out in $9.5 \%$ of cases. Although all postoperative adjustments were carried out at the surgeon's discretion, the difference between the number of postoperative adjustments in the DS and TRAB groups seemed to result from differences between the two surgical techniques rather than between the management decisions taken by the two surgeons. TRAB is a penetrative surgery and, therefore, does not allow needling with antimitotic agents or goniopuncture. These results are consistent with those for DS in uveitic glaucoma reported by Auer ${ }^{9}$ who carried out needling in 5 of 14 eyes (36\%) and goniopuncture in 4 of 14 (29\%).

Moreover, Ambresin ${ }^{15}$ observed that goniopuncture was also necessary after DS in non-uveitic glaucoma, in 9 out of 20 eyes (45\%), and 5-FU needling, in 7 out of 20 eyes $(35 \%)$. In a recent prospective study, Obeidan ${ }^{10}$ observed the need for goniopuncture in 2 out of 13 patients (15\%). In this study, the high success rate of DS-treated eyes was attributable to the close postoperative follow-up, which ensured that interventions to lower IOP were carried out without delay.

Although preoperative flare values were not different in the two groups, the mean preoperative value was higher in the TRAB group. In this connection, juvenile idiopathic arthritis (JIA) was more frequent in the TRAB group ( 5 eyes $v s 1$ eye in the DS group, $P=0.089$ ), and it has been reported that JIA-associated uveitis may create a large blood-aqueous barrier breakdown, with a positive correlation between increased laser flare photometry values and complications at baseline in 
children with this type of uveitis. ${ }^{19}$ Here, this feature might have increased the rate of failure in our TRAB group. However, although intraocular inflammation at postoperative day 7 was more marked in the TRAB than the DS group (as reported by Chiou in non-uveitic glaucoma ${ }^{20}$ ) in this study, final visual acuity and the complications rate were not different in the two groups.

A limiting factor was the dropout rate of approximately $30 \%$ for the mid-term follow-up examination after surgery because of the retrospective nature of the study. However, the total strength of the patients included was high, because uveitic glaucomas are much less frequent than primary open-angle glaucomas. The number of eyes treated in our study (41) was larger than in most other investigations of uveitic glaucomas (for instance, Ho reported 31 eyes, ${ }^{21}$ Auer, $14,{ }^{9}$ Ozdal, $19^{22}$ and Obeidan, $13^{10}$ ), but was similar to the 44 and 40 eyes in the series studied by Ceballos $e a^{1}$ and Molteno et al., ${ }^{3}$ respectively.

Another limiting factor in this investigation was its retrospective character. However, there was no choice of surgical technique for individual patients, as they were recruited serially by the two surgeons, each of whom is a specialist of DS or TRAB.

In conclusion, although randomised prospective comparative studies of these two procedures are still necessary, our results suggest that DS with an implant and TRAB with antiproliferative agents, are both effective for the management of uveitic glaucoma. They result in equal control of IOP, with few postoperative complications. The DS generates less inflammation during the early postoperative follow-up, but requires close monitoring for appropriate adjustment of pressure-lowering interventions, such as goniopuncture or needling. TRAB, on the other hand, leads to high transient postoperative inflammation, but allows direct IOP reduction with very few postoperative adjustments, and might therefore be indicated in cases, in which close monitoring is difficult.

\section{References}

1 Ceballos EM, Beck AD, Lynn MJ. Trabeculectomy with antiproliferative agents in uveitic glaucoma. J Glaucoma 2002; 11: 189-196.

2 Towler HM, McCluskey P, Shaer B, Lightman S. Long-term follow-up of trabeculectomy with intraoperative 5-fluorouracil for uveitis-related glaucoma. Ophthalmology 2000; 107: 1822-1828.

3 Molteno AC, Sayawat N, Herbison P. Otago glaucoma surgery outcome study: long-term results of uveitis with secondary glaucoma drained by Molteno implants. Ophthalmology 2001; 108: 605-613.

4 Mermoud A. [Deep sclerectomy: surgical technique]. J Fr Ophtalmol 1999; 22: 781-786.
5 Mermoud A, Schnyder CC, Sickenberg M, Chiou AG, Hédiguer SE, Faggioni R. Comparison of deep sclerectomy with collagen implant and trabeculectomy in open-angle glaucoma. J Cataract Refract Surg 1999; 25: 323-331.

6 Cillino S, Di Pace F, Casuccio A, Cillino G, Lodato G. Deep sclerectomy $v$ s trabeculectomy with low-dosage mitomycin C: four-year follow-up. Ophthalmologica 2008; 222: 81-87.

7 Sarodia U, Shaarawy T, Barton K. Nonpenetrating glaucoma surgery: a critical evaluation. Curr Opin Ophthalmol 2007; 18: 152-158.

8 Novak-Laus K, iæ Z, Ivekovic R, Korsic J, Tedeschi-Reiner E, Masnec-Paskvalin S et al. Trabeculectomy with mitomycin C in glaucoma associated with uveitis. Coll Antropol 2005; 29(Suppl 1): 17-20.

9 Auer C, Mermoud A, Herbort CP. Deep sclerectomy for the management of uncontrolled uveitic glaucoma: preliminary data. Klin Monatsbl Augenheilkd 2004; 221: 339-342.

10 Al Obeidan SA, Osman EA, Al-Muammar AM, Abu El-Asrar AM. Efficacy and safety of deep sclerectomy in uveitic glaucoma. Int Ophthalmol 2008. [E-pub ahead of print].

11 Arruabarrena C, Munoz-Negrete FJ, Marquez C, Rebolleda G. [Results of nonpenetrating deep sclerectomy in inflammatory glaucoma: one year follow up]. Arch Soc Esp Oftalmol 2007; 82: 483-487.

12 Hill RA, Nguyen QH, Baerveldt G, Forster DJ, Minckler DS, Rao N et al. Trabeculectomy and Molteno implantation for glaucomas associated with uveitis. Ophthalmology 1993; 100: 903-908.

13 Miserocchi E, Carassa RG, Bettin P, Brancato R. Viscocanalostomy in patients with glaucoma secondary to uveitis: preliminary report. J Cataract Refract Surg 2004; 30: 566-570.

14 Wilson MR, Mendis U, Paliwal A, Haynatzka V. Long-term follow-up of primary glaucoma surgery with Ahmed glaucoma valve implant $v$ s trabeculectomy. Am J Ophthalmol 2003; 136: 464-470.

15 Ambresin A, Shaarawy T, Mermoud A. Deep sclerectomy with collagen implant in one eye compared with trabeculectomy in the other eye of the same patient. J Glaucoma 2002; 11: 214-220.

16 El Sayyad F, Helal M, El-Kholify H, Khalil M, El-Maghraby A. Nonpenetrating deep sclerectomy vs trabeculectomy in bilateral primary open-angle glaucoma. Ophthalmology 2000; 107: 1671-1674.

17 Souissi K, El Afrit MA, Trojet S, Kraiem A. [Deep sclerectomy for the management of uveitic glaucoma]. J Fr Ophtalmol 2006; 29: 265-268.

18 Shaarawy T, Mermoud A. Deep sclerectomy in one eye $v s$ deep sclerectomy with collagen implant in the contralateral eye of the same patient: long-term follow-up. Eye 2005; 19: 298-302.

19 Davis JL, Dacanay LM, Holland GN, Berrocal AM, Giese MJ, Feuer WJ. Laser flare photometry and complications of chronic uveitis in children. Am J Ophthalmol 2003; 135: 763-771.

20 Chiou AG, Mermoud A, Jewelewicz DA. Post-operative inflammation following deep sclerectomy with collagen implant vs standard trabeculectomy. Graefes Arch Clin Exp Ophthalmol 1998; 236: 593-596.

21 Ho CL, Walton DS. Goniosurgery for glaucoma secondary to chronic anterior uveitis: prognostic factors and surgical technique. J Glaucoma 2004; 13: 445-449.

22 Ozdal PC, Vianna RN, Deschenes J. Ahmed valve implantation in glaucoma secondary to chronic uveitis. Eye 2006; 20: 178-183. 